\title{
Comparative Evaluation of Three Wireless Sensor Network Transceivers in a High Radiation Environment
}

\author{
Q. Huang, Member, IEEE, J. Jiang*, Fellow, IEEE and Y. Q. Deng, Student Member, IEEE \\ University of Western Ontario \\ *jjiang@uwo.ca
}

\begin{abstract}
This paper presents on the results of radiation studies for three commonly used wireless sensor nodes based on the following protocols: ZigBee, WirelessHART, ISA 100.11a, and network devices built with commercial off-the-shelf (COTS) components. The level of radiation considered is at par with that experienced at Fukushima Daiichi Nuclear Power Plant after the accident. An experimental setup is developed to monitor behaviors of each wireless device and network real-time under the ${ }^{60} \mathrm{Co}$ gamma radiator at The Ohio State University Nuclear Reactor Lab (OSU-NRL). The experimental results have indicated that the performance of the communication channels and wireless signal parameters do not degrade significant under such radiation. However, all the tested devices and networks can only survive for several hours under the high dose rate condition $(20 \mathrm{~K} \mathrm{Rad} / \mathrm{h})$. The results of these experimental studies have provided useful references to those who design and manufacture COTS-based wireless monitoring systems for use in high level radiation environments.
\end{abstract}

Index Terms - Total dose test, industrial wireless sensor networks, rad-hardened, severe accident, nuclear power plant

\section{INTRODUCTION}

One of the lessons from Fukushima Daiichi nuclear disaster is the difficulty to gain up-to-date status about the key plant status, such as water levels in spent fuel bays, hydrogen concentration in reactor buildings, and temperatures in heat transport systems, after the accident due to loss of on-site power for monitoring instruments and strong radiation [1]. Considering various potential scenarios during a severe accident, a potential approach is to use wireless technologies to implement post-accident monitoring system (PAMS) which can provide much needed information about the plant conditions without relying on likely damaged communication infrastructure [1-3].

In past decades, a number of wireless transceivers and sensor networks have been developed to acquire data in a wide variety of applications [4-7]. A designer of industrial wireless systems has several wireless transceivers and network standards to choose from. However, in the event of a severe nuclear accident, a significant amount of radiation can be released due to failure of protection layers [8], to minimize the impact of radiation on these wireless systems, more considerations have to be given during component and protocol selection process for these monitoring instruments.
Taking Fukushima accident as an example, in March 2012, the level of radiation is estimated to up to $73 \mathrm{~Sv} / \mathrm{h}$ inside the containment of No.2 reactor [9], and Feb. 2017, the level has shot up to $530 \mathrm{~Sv} / \mathrm{h}[10]$. Such high level of radiation can, no doubt, cause severe damage to wireless transceivers and networks if no precautions are taken. Before any protection measures can be taken, it is necessary to investigate how different wireless devices and network components react to radiation under similar radiation conditions. Unfortunately, there are only limited resources available on radiation responses of industrial wireless devices and networks in the literature. To fill this gap, the characteristics of commonly used industrial wireless devices and networks are studied. The results of this study provides a scientific basis in the selection of suitable wireless technologies and network devices in such applications.

Based on those studies and analysis, six industrial wireless nodes built with commercial off-the-shelf(COTS) components, CC2530 (2.4 GHz ZigBee standard), CC2520 (2.4 GHz WirelessHART standard), CC2530 (2.4 GHz ISA100.11a standard), are chosen to undergo irradiation tests in a gamma radiation environment. Since these are destructive tests, a group of diversified wireless devices are therefore built using different industrial wireless networks for the radiation tests. A total dose test is performed to measure the behaviors of the developed wireless devices and networks by directly exposing them to 60Co gamma irradiator at The Ohio State University Nuclear Reactor Laboratory (OSU-NRL) under a high dose rate $(20 \mathrm{~K}$ $\mathrm{Rad} / \mathrm{h}$ ). The results of this test will provide guidelines to design wireless monitoring instruments for using in high level radiation environments. They serve as important references to assist other researchers and engineers to design and manufacture radiation-hardened (rad-hardened) monitoring system by using COTS components.

The reminder of the paper is organized as follows. The basics of the three industrial wireless devices and network standards are described in Section II. Subsequently, the chosen wireless sensor nodes and network devices are explained in Section III. Section IV presents experimental setups and summarizes the results with analysis. Finally, a number of observations are made and conclusions are drawn in Section V.

\section{INDUSTRIAL WIRELESS DEVICES AND NETWORKS}

Wireless sensor network technologies have been used in many industrial applications, such as safety systems, control systems, and monitoring systems [4-6]. In a typical wireless 
sensor network system, a measured process variable from a sensor is transmitted from one node to another or to a base station using radio frequency (RF) waveforms. Several distinct frequency bands have been dedicated to wireless sensor networks for industrial applications based on industry and governmental regulations. They are, for example, at $433 \mathrm{MHz}$, $868 \mathrm{MHz}, 915 \mathrm{MHz}$, and $2.4 \mathrm{GHz}$.

In the past few decades, a number of wireless transceivers have been developed with various communication functions. On the other hand, various functions and network protocols for sensor nodes have been defined in wireless sensor network standards for integration with the networks. Recently, several wireless sensor network standards based on the IEEE 802.15.4 have also been developed, such as ZigBee, WirelessHART, and ISA100.11a [4-6]. Brief description of these protocols are in order.

In general, radiation particles lose their energy through either non-ionization processes (displacement damage) and/or ionization processes when they pass through semiconductor devices. Radiation effects on electronics can generally be classified into three categories:

ZigBee: ZigBee network supports Star, Tree, and Mesh topologies, as shown in Fig. 1. Star topology is often choice for industrial applications. Within the network, the roles that the devices play can be categorized into the following: (1) Coordinator, which is used to initialize, maintain, and control the network; (2) Router, which is responsible for routing messages; and (3) End Device, which executes the intended functions associated with the application [5].

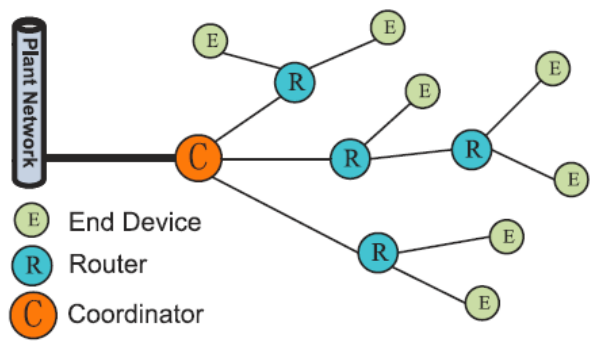

Fig. 1 ZigBee network [5]

WirelessHART: WirelessHART supports both Star and Mesh network topology as shown in Fig. 2. Basic elements in a WirelessHART network include: Field Devices, Gateway, Access Point, Network Manager, and Handheld Devices [5]. A sensor for measuring process variables is fed to the Field Device for transmission. An Access Point is used to connect the Field Devices to the Gateway. The network manager can be used to configure the network, schedule and overseeing communications among different devices[5]. WirelessHART systems have found many industry applications [6].

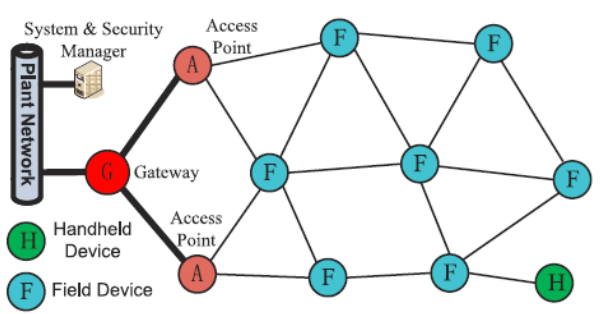

Fig. 2 WirelessHART network [5]

(3) ISA100.11a: ISA100.11a supports both Mesh and Star network topology as shown in Fig. 3. A typical ISA100.11a network includes two type devices: Field Devices and Infrastructure Devices. The former includes Routing Devices, I/O Devices, and Handheld Devices. The latter includes Backbone Routers, Gateways, and System and Security Managers [5]. ISA100.11a network has been shown to be a robust and secure communication system for industrial applications [6].

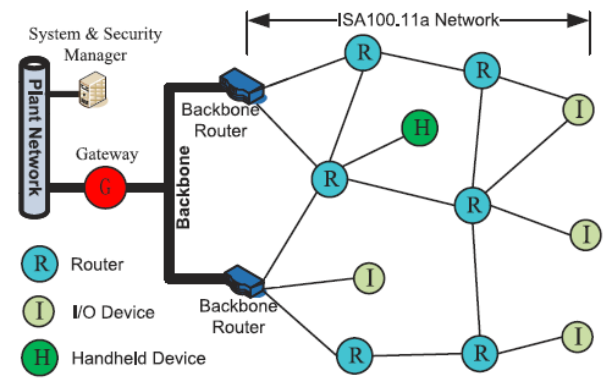

Fig. 3 ISA100.11a network [5]

\section{DEVElopment OF DEVICES FOR RADIATION TESTS}

For radiation experiments for total ionizing dose conditions, three group of wireless devices, labelled as Sample\#1 to Sample\#3, have been designed and produced using two different transceivers and controller units. All three systems are operating at $2.4 \mathrm{GHz}$. The details are summarized in TABLE I:

TABLE I: WIRELESS DEVICES USED IN THE IRRADIATION TESTS

\begin{tabular}{|c|c|c|c|}
\hline Sample ID & Transceivers & Protocols & Controller Units \\
\hline Sample \#1 & CC2530 & ZigBee & AVR ATMEGA 328 \\
\hline Sample \#2 & CC2520 & WirelessHART & Proprietary \\
\hline Sample \#3 & CC2530 & ISA100.11a & STM32L486 \\
\hline
\end{tabular}

These sample circuit boards are shown in Fig.4. The radiation tests are carried out when the circuit boards are on power, and the wireless networks have also been setup to relay the response of the tested wireless device out of the test rig and send to a Control Workstation through the RS232 protocol. The performance of each device is being monitored continually throughout the tests until it ceases operation. The logged data can then be used for determination of potential causes. 


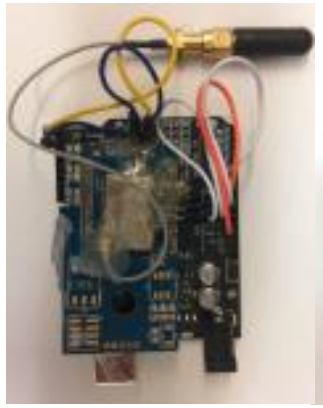

Sample\#1

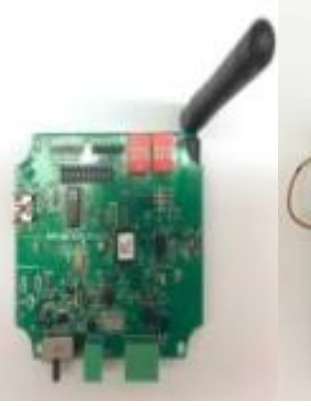

Sample\#2

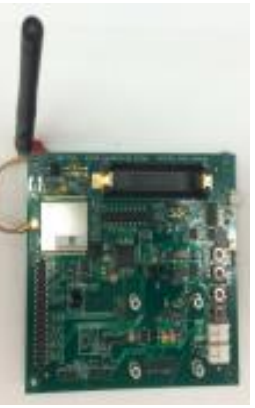

Sample\#3
Fig. 4. Samples used in the total dose tests

For Sample\#1, a star ZigBee network has been setup using E800-DTU (Z2530-485-20) as the Router and Coordinator and E18-MS1PA1-IPX as the transceiver for the End Device. Both are from Ebyte Electronic Company. Furthermore, AVR ATMEGA 328 is used as the controller for the End Device. End Device sends one message to the Router and Coordinator every two seconds.

For WirelessHART system of Sample\#2, the Field Device used in the experiments is Awia Warrior 220 manufactured by AwiaTech Company. It communicates to the WirelessHART Gateway. The HART Server installed on the Control Workstation is used to configure the WirelessHART network.

In case of ISA100.11a system, i.e. Sample\#3, the transceiver used as the Field Router and IO Device is VN210 from Nivis. The network is formed using VersaRouter 900 as the Backbone Router and Gateway which also perform the functions of System Manager, Security Manager, and System Time Source. A web application is developed to remotely check and configure the ISA100.11a network.

\section{FACILITIES AND ENVIRONMENT FOR RADIATION TESTS}

The radiation source is a $60 \mathrm{Co}$ irradiator tube at the OSUNRL as illustrated in Fig. 5. The samples are placed in the 6inch tube one at a time. A shielding elevator delivers the test sample to the irradiation location. The peak dose rate is estimated to be at $20 \mathrm{~K} \mathrm{Rad} / \mathrm{h}$. The samples are powered through cables from a $+5 \mathrm{v}$ power supply and a DC-DC Converter, which are located outside the irradiator. Several equipment, such as oscilloscope and spectrum analyzer, is used to measure electronic signals and communication channel performance.

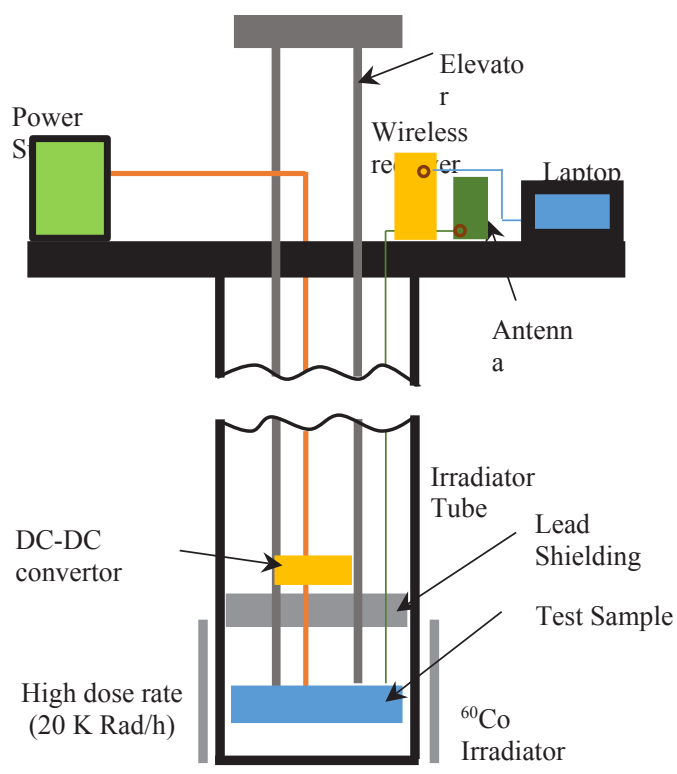

Fig. 5. Schematic of the experimental irradiator tube

The actual test environment is shown in Fig. 6 with all the facilities. It is important to point out that each test only involves one sample. Furthermore, the signal for the antenna of the wireless devices are routed outside before connecting to an antenna to avoid EM shielding of the test tube.

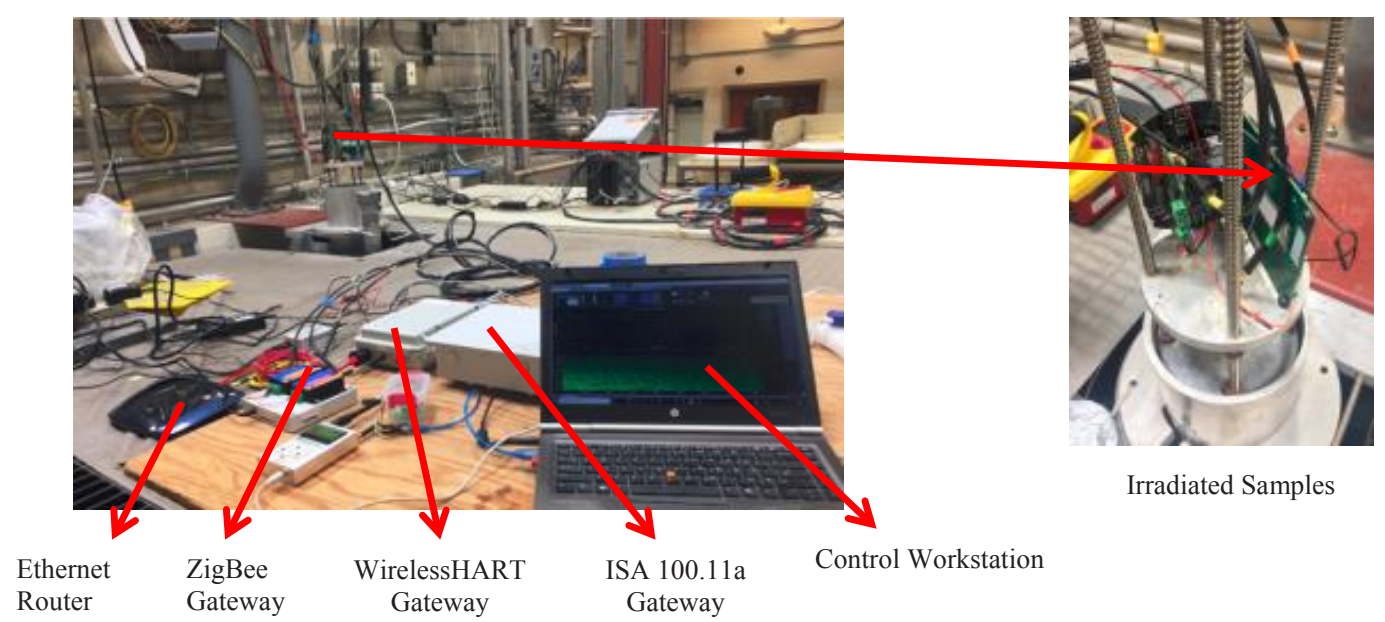

Fig. 6 Actual test environment for ZigBee, WirelessHART, and ISA100.11a devices 
During each test, the performance of the communication channels and wireless signals are continuously monitored to capture the following performance indicators: loss packet rate, error packet rate, shifts in frequency, and Received Signal Strength Indicator (RSSI). The irradiation test continues as long as the device is functioning and transmitting wireless signals. When the device ceases operation and exact time of breakdown is recorded. Hence, the survival time of the device under a dose rate of $20 \mathrm{~K} \mathrm{Rad} / \mathrm{h}$ can be obtained. The total dosage (TD) of ionizing radiation deposited on to the wireless device can be calculated as:

$$
=20 \mathrm{~K} \mathrm{Rad} / \mathrm{h} \times \text { survival time }
$$

This variable represents how much total radiation a device can take before giving in and lead to a failure.

\section{EXPERIMENTAL RESULTS}

After three destructive tests, the survival times for the three samples are recorded. The results are summarized in TABLE II.
TABLE II: RADIATION TEST RESULTS

\begin{tabular}{|c|c|c|c|}
\hline & Sample\#1 & Sample\#2 & Sample\#3 \\
\hline Survival time (h) & $192 \mathrm{~m}$ & $50 \mathrm{~m}$ & $62 \mathrm{~m}$ \\
\hline First failed component & $\begin{array}{c}\text { CPU and } \\
\text { Transceiver }\end{array}$ & Transceiver & Transceiver \\
\hline $\begin{array}{c}\text { Total Dosage (TD) before } \\
\text { the first failure (K Rad) }\end{array}$ & 64 & 16.7 & 20.0 \\
\hline
\end{tabular}

It can be observed from TABLE II that the ZigBee (Sample\#1) device has the best performance in terms of radiation tolerance, as it survives the radiation environment much longer than the other two devices. This is followed by the ISA100.11a. However, when ZigBee device fails, both CPU and Transceiver have failed, but for the other two devices, the weakest component is the Transceiver.

To investigate how radiation affects the quality of the communication systems, two type of tests have been carried out: one is in an ideal laboratory environment free from ionizing radiation. These results provide a reference for comparison and the other is on-line and real-time measurements while the devices are at the bottom of the test tube. The results for Sample\#1 to Sample\#3 are summarized in TABLE III to V, respectively.

TABLE III

COMMUNICATION PERFORMANCE OF ZIGBEE DEVICE AND NETWORK UNDER NORMAL AND HIGH DOSE RATE CONDITIONS

\begin{tabular}{|c|c|c|c|c|c|c|c|c|}
\hline $\begin{array}{l}\text { Radiation } \\
\text { condition } \\
\end{array}$ & $\begin{array}{c}\text { Duration } \\
\text { (minutes) }\end{array}$ & $\begin{array}{c}\text { Total } \\
\text { packets }\end{array}$ & $\begin{array}{c}\text { Loss } \\
\text { packets }\end{array}$ & $\begin{array}{c}\text { Loss rate } \\
(\%)\end{array}$ & $\begin{array}{c}\text { Error } \\
\text { packet }\end{array}$ & $\begin{array}{c}\text { Error rate } \\
(\%)\end{array}$ & $\begin{array}{c}\text { Frequency } \\
(\mathrm{GHz})\end{array}$ & $\begin{array}{r}\text { RSSI } \\
(\mathrm{dBm})\end{array}$ \\
\hline \multirow{5}{*}{$\begin{array}{l}\text { Normal } \\
\text { condition }\end{array}$} & 30 & 150 & 0 & 0.00 & 0 & 0.00 & 2.405 & -43.00 \\
\hline & 60 & 150 & 0 & 0.00 & 0 & 0.00 & 2.406 & -32.00 \\
\hline & 90 & 150 & 0 & 0.00 & 0 & 0.00 & 2.407 & -53.50 \\
\hline & 150 & 150 & 0 & 0.00 & 0 & 0.00 & 2.404 & -35.00 \\
\hline & 180 & 150 & 0 & 0.00 & 0 & 0.00 & 2.406 & -48.00 \\
\hline \multirow{6}{*}{$20 \mathrm{~K} \mathrm{Rad} / \mathrm{h}$} & 30 & 150 & 0 & 0.00 & 0 & 0.00 & 2.411 & -22.50 \\
\hline & 60 & 150 & 0 & 0.00 & 0 & 0.00 & 2.411 & -53.50 \\
\hline & 90 & 149 & 1 & 0.67 & 0 & 0.00 & 2.410 & -45.50 \\
\hline & 150 & 150 & 0 & 0 & 0 & 0.00 & 2.409 & -35.50 \\
\hline & 180 & 150 & 0 & 0 & 0 & 0.00 & 2.409 & -39.50 \\
\hline & 192 & & & & Fail & & & \\
\hline
\end{tabular}

TABLE IV

COMMUNICATION PERFORMANCE OF WIRELESSHART DEVICE AND NETWORK UNDER NORMAL AND HIGH DOSE RATE CONDITIONS

\begin{tabular}{ccccccc}
\hline \hline $\begin{array}{c}\text { Radiation } \\
\text { condition }\end{array}$ & $\begin{array}{c}\text { Duration } \\
(\text { minutes })\end{array}$ & Device ID & $\begin{array}{c}\text { Analog value } \\
(\mathrm{mA})\end{array}$ & $\begin{array}{c}\text { Percent } \\
\text { range }\end{array}$ & $\begin{array}{c}\text { Frequency } \\
(\mathrm{GHz})\end{array}$ & $\begin{array}{c}\text { RSSI } \\
(\mathrm{dBm})\end{array}$ \\
\hline \multirow{2}{*}{$\begin{array}{c}\text { Normal } \\
\text { condition }\end{array}$} & 30 & 131874 & 11.90 & $49.50 \%$ & 2.402 & -49.00 \\
\cline { 2 - 7 } & 60 & 131874 & 11.90 & $49.50 \%$ & 2.407 & -48.50 \\
\hline \multirow{3}{*}{$20 \mathrm{~K} \mathrm{Rad} / \mathrm{h}$} & 30 & 131874 & 11.90 & $49.50 \%$ & 2.402 & -48.00 \\
\cline { 2 - 7 } & 50 & 131874 & 11.90 & $49.50 \%$ & - & - \\
\cline { 2 - 7 } & 51 & \multicolumn{5}{c}{ Fail } \\
\hline \hline
\end{tabular}

TABLE V

COMMUNICATION PERFORMANCE OF ISA100.11A DEVICE AND NETWORK UNDER BOTH A NORMAL CONDITION AND A HIGH DOSE RATE CONDITION

\begin{tabular}{ccccccccc}
\hline \hline $\begin{array}{c}\text { Radiation } \\
\text { condition }\end{array}$ & $\begin{array}{c}\text { Duration } \\
(\text { minutes })\end{array}$ & $\begin{array}{c}\text { Total } \\
\text { packets }\end{array}$ & $\begin{array}{c}\text { Loss } \\
\text { packets }\end{array}$ & $\begin{array}{c}\text { Loss rate } \\
(\%)\end{array}$ & $\begin{array}{c}\text { Error } \\
\text { packet }\end{array}$ & $\begin{array}{c}\text { Error rate } \\
(\%)\end{array}$ & $\begin{array}{c}\text { Frequency } \\
(\mathrm{GHz})\end{array}$ & $\begin{array}{c}\text { RSSI } \\
(\mathrm{dBm})\end{array}$ \\
\hline \multirow{2}{*}{$\begin{array}{c}\text { Normal } \\
\text { condition }\end{array}$} & 30 & 119 & 0 & 0.00 & 0 & 0.00 & 2.406 \\
\hline & 60 & 1375 & 0 & 0.00 & 0 & 0.00 & 2.403 \\
\hline
\end{tabular}


As can be seen that radiation has hardly any effects to the performance of communication quality for both ZigBee and WirelessHART devices. For the ISA100.11a device, there are some minor loss of packets under high dose conditions, which can be easily dealt with through error correction codes.

As far as the quality of wireless signals is concerned, no effects have been observed on the central operating frequency of $2.4 \mathrm{GHz}$. However, fluctuations of RSSI are observed. Unfortunately, it is not conclusive to link such variations to the radiation, as there is no obvious trend observed from the data.

From a hardware and software complexity point of view, WirelessHART and ISA100.11a contain more hardware components and software functions, some may be more susceptible to radiation damage. On the other hand, ZigBee is relatively simpler in both hardware and software design. It turns out to be more tolerant to radiation.

Another important point to note is that none of these devices should be put in use for a severe accident monitoring system without proper protection and radiationtolerant design, since they can be easily damaged in a high level radiation environment.

\section{CONCLUSION}

To examine the suitability of using industrial wireless sensor network devices in a high level radiation environment, three most commonly used wireless sensor network devices, ZigBee, WirelessHART, and ISA100.11a, have been tested in a radiation environment during on-power operations. A $60 \mathrm{Co}$ gamma radiation source is used to provide a consistent radiation environment. The operating conditions of key components on the devices are monitored continuously so that the first failed component can be identified in the post-analysis. It is interesting to observe that the wireless device based on ZigBee protocol has the strongest radiation tolerance, while WirelessHART and ISA100.11a devices share similar outcomes. A very important conclusion is that these devices can only survive for a limited amount of time, so much shorter than needed for use in a monitoring system for severe accident. Hence, adequate protections against total radiation exposure have to be taken either through shielding or redundant design. This study provides a useful reference for the design of wireless devices and networks to be deployed in high level radiation environments.

\section{ACKNOWLEDGMENT}

This work is financially supported by the University Network of Excellence in Nuclear Engineering (UNENE) and the Natural Sciences and Engineering Research Council of Canada (NSERC). Authors would like to acknowledge technical support from the staff at The Ohio State University Nuclear Reactor Laboratory (NRL) for their assistance during the experiments.

\section{REFERENCES}

[1] K. Nagatani, S. Kiribayashi, Y. Okada, K. Otake, K. Yoshida, S. Tadokoro, T. Nishimura, T. Yoshima, and E. Koyanagi. Emergency response to the nuclear accident at the Fukushima Daiichi nuclear power plants using mobile rescue robots. Journal of Field Robotics, vol. 30, pp. 44-63, 2013.

[2] M. Andreeva, M.P. Pavlova, and P.P Groudev, "Overview of plant specific severe accident management strategies for Kozloduy nuclear power plant, WWER1000/320," Annals of Nuclear Energy, vol. 35, pp. 555$564,2008$.

[3] H. Kinoshita, R. Tayama, Y. Kometani, and Y. Kani, "Development of new technology for Fukushima Daiichi nuclear power station reconstruction," Hitachi Review, vol. 63, pp. 41-46, 2014.

[4] J. Yick, B. Mukherjee, and D. Ghosal. Wireless sensor network survey. Computer Networks, vol. 52, pp. 22922330, 2008.

[5] Q. Wang, and J. Jiang. Comparative examination on architecture and protocol of industrial wireless sensor network standards. IEEE Communication Survey \& Tutorials, vol. 18, pp. 2197-2219, 2016.

[6] A. Ajith Kumar S., K. Øvsthus and L.M. Kristensen, An industrial perspective on wireless sensor networks - a survey of requirements, protocols, and challenges. IEEE Communications Surveys \& Tutorials, vol. 16, pp. 13911412, 2014.

[7] O. Georgiou, and U. Raza. Low power wide area network analysis, Can LoRa Scale? IEEE Wireless Communications Letters, vol. 6, pp. 162-165, 2017.

[8] A.A. Adalja, E.S. Toner, A. Cicero, J. Fitzgerald, and T.V. Inglesby, Radiation at Fukushima: basic issues and concepts. Clinicians' Biosecurity News, Analysis of Advances and Challenges in Clinical Biosecurity.

[9] R. Eisler, The Fukushima 2011 disaster. U.S. Geological Survey, 2012.

[10][Online] Available: The Fukushima nuclear reactor radiation at highest level since 2011 meltdown, https://www.theguardian.com/environment/2017/feb/03/f ukushima-daiichi-radiation-levels-highest-since-2011meltdown. Access date: Feb. 3, 2017. 